\title{
Wind Tunnel Investigation of Shapes for Balloon Shelters ${ }^{1}$
}

\author{
RoBert N. MERoney \\ Civil Engineering Department, Colorado State University, Fort Collins 80521 \\ AND Erich J. Plate \\ Institut Wasser Bau III, Universität Karlsruhe, Germany \\ (Manuscript received 6 March 1972)
}

\section{ABSTRACT}

\begin{abstract}
In support of a balloon shelter development program a series of wind tunnel tests were performed. The study concerned the scaling criteria for such a simulation; velocity, turbulence and frequency spectra downwind of four basic shelter shapes; the effect of screen material on shelter efficiency; and the influence of a simulated balloon presence upon the effectiveness of the shelter.
\end{abstract}

\section{Introduction}

A wind tunnel and field investigation were performed on possible launch shelters for meteorological balloons. The purpose of the effort was to aid in the design of a screen which would reduce the wind from $15 \mathrm{kt}$ to below $8 \mathrm{kt}$ over a volume sufficient to assist with inflation and launching of large balloons ( $\sim 70 \mathrm{ft}$ high $\times 70 \mathrm{ft}$ wide $\times 70 \mathrm{ft}$ deep). (See Fig. 1.) The emphasis of the study was to determine the effects of basic shelter configurations and screen materials upon the sheltered area.

The program was organized to examine the following aspects of the shelter dynamics:

Height, length and breadth of the sheltered areas for various shelter configurations.

Comparative effectiveness of a solid shelter compared with various porous screen materials.

Quantitative measurement of the frequency and intensity of gusts shed from the upper edge and sides of the proposed shelter configurations.

The effect of changes in the included angle on sheltered area and gusting.

The effect of the presence of a balloon shape within the sheltered area upon the effectiveness of the shelter.

Four basic shelter shapes were tested in the wind tunnel at a scale of 1:70. Various shelter angles, screen materials, and the effect of the presence of a partially inflated balloon were examined. Wind velocities were subsequently measured behind a 1:7 scale model in the atmosphere for one shelter angle and two screen materials.

\footnotetext{
${ }^{1}$ Work supported by the National Center of Atmospheric Research, Boulder, Colo., under subcontract NCAR-75-68.
}

\section{General considerations of similarity and the fluid dynamics of wind breaks}

In some earlier work (Plate and Lin, 1965) it is shown that modeling of a field wind break situation in a laboratory is accomplished if $C_{D}$ (the drag coefficient of the shelter) and the ratio $h / \delta$ are the same in both field and laboratory, where the length $h$ is the structure height and $\delta$ the thickness of the boundary layer. Although these requirements were for two-dimensional flow fields, it can be expected that only minor modification would be required for the three-dimensional counterpart.

Constant drag coefficients $C_{D}$ can be obtained approximately by having sharp edges of the shelters both in model and prototype. Then the drag coefficient

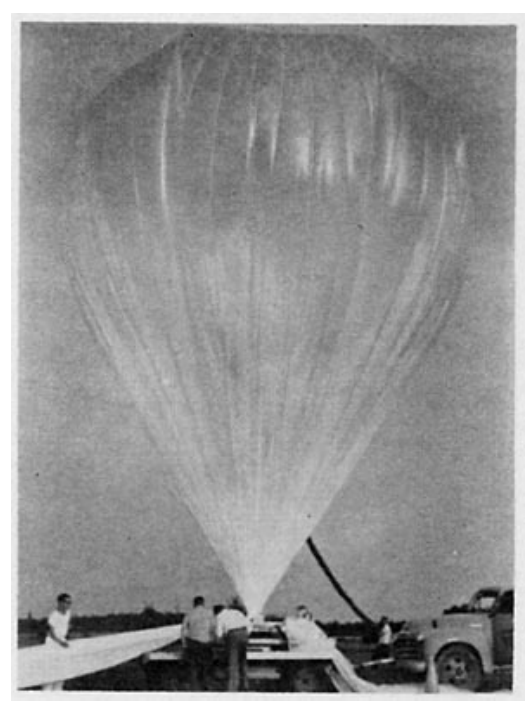

FIG. 1. Experimental balloon inflated and ready for launch. 
defined by

$$
C_{D}=\frac{D}{\frac{1}{2} \rho u_{\infty}{ }^{2} h w},
$$

where $D$, the drag on the shelter, becomes independent of the Reynolds number $\operatorname{Re}=u_{\infty} h / \nu$. In this equation, $h$ is the height and $w$ the breadth of the projection of the shelter on a plane perpendicular to the direction of the ambient air flow $u_{\infty}$ (at some reference height). Ordinarily $C_{D}$ would be a function of Reynolds number. However, by sharpening the edges of the shelter, the separation line of the boundary layer on the shelter becomes fixed, resulting in a $C_{D}$ which is independent of the Reynolds number. It does, however, depend slightly on $h / \delta$, but this dependency is not critical and can be adjusted for by making the boundary layer of the approach flow as thick as possible.

The drag coefficient not only determines the drag on the shelter but also the shape of the flow field downstream from the shelter. In general, the larger $C_{D}$, the larger will be the sheltered area, but evidently at the price of a larger drag force, as well as higher turbulence levels.

For a solid screen, or a square flat plate, it is possible to obtain the drag coefficient, to a first approximation, from the relation

$$
\begin{aligned}
& \left.\frac{\left.C_{D} \text { (rectangular plate }\right)}{\left.C_{D} \text { (infinite } 2 d \text { plate }\right)}\right|_{\text {in boundary layer }} \\
& =\left.\frac{C_{D}(\text { rectangular plate })}{\left.C_{D} \text { (infinite } 2 d \text { plate }\right)}\right|_{\text {in free stream }},
\end{aligned}
$$

or

$$
\frac{\left.C_{D} \text { (rectangular plate }\right)}{0.8}=\frac{1.16}{1.90},
$$

where the free-stream values are from Rouse $(1950)$ and the value of 0.8 for the drag coefficient of the infinite plate in a boundary layer has been taken from experimental results of Plate (1964). Consequently $C_{D}=0.5$ to a first approximation.

Some measurements of Vickery (1968) for a plate which was neither fully in the free stream nor on a floor were found to yield $C_{D}=1.0$ which falls between the assumed free-stream value of 1.16 and the calculated boundary layer value of 0.5 . A safe value, to be used in calculation, might therefore be taken as about $C_{D}=0.7$.

In the quoted paper, Vickery also points out that in addition to the mean drag, there also occurs a fluctuating drag whose rms value might be as much as $10 \%$ of the mean. He does not give a peak value, but a suitable safety factor should be used. In view of the fact that the structure of the shelter will be very light, a safety factor of at least 2 is recommended, i.e., for the design of the structure, $C_{D}=1.0-1.2$ should be used.

It is very likely that the effect of porosity is also a Reynolds number effect, but this time the Reynolds number should be based on the properties of the screen material. Since air flow and viscosity in model and prototype are the same, it is required that the screens be the same also, to meet Reynolds number similarity. Actually, however, it is found that for a given screen material the aerodynamic behavior is practically independent of Reynolds number. A measure of the aerodynamic behavior can be obtained by determining the pressure drop $\Delta p$ across a screen which passes a velocity of $\bar{u} \mathrm{ft} \mathrm{sec}{ }^{-1}$. The pressure drop coefficient,

$$
c_{p}=\frac{\Delta p}{\frac{1}{2} \rho \bar{u}^{2}}
$$

should become independent of the Reynolds number.

For a porous screen, the pressure drop coefficient yields a measure of the force exerted on the screen. Let $\bar{u}$ be the velocity observed, in the model case directly downstream of the screen. Then, to a rough approximation, we have

$$
D=c_{p} \frac{1}{2} \rho \bar{u}^{2} w h
$$

or, if the reduction factor $c$ is introduced, i.e.,

$$
c=\frac{\bar{u}}{u_{\infty}}
$$

which signifies the reduction of velocity obtained by a screen, then

$$
D=c_{p} c^{2 \frac{1}{2} \rho u_{\infty}{ }^{2} r \omega h} .
$$

For a given screen material and shelter shape, the coefficients $c_{p}$ and $c$ are found from wind tunnel experiments.

Comparison of Eqs. (1) and (7) shows that for a porous screen we have

$$
C_{D}=c_{p} c^{2}
$$

The experiments show that for a porous screen, both $c$ and $c_{p}$ are approximately independent of velocity, so that $C_{D}$ is found independent of Reynolds number for porous screens as well-provided that the screens are the same in model and prototype.

For the bug screen material used, we find a value of $c_{p}=0.62$ and a reduction factor $c=0.5$. Consequently, the equivalent drag coefficient, according to Eq. (8), is

$$
C_{D}=0.62 \frac{1}{4}=0.16 \text {. }
$$

It goes without saying that (8) is valid only for $C_{D}<0.5-0.7$. Once $C_{D}=0.5-0.7$ is reached, a screen behaves like a solid screen regardless of its actual porosity.

The parameter $h / \delta$ determines mainly the velocity distribution downstream of the shelter, outside the sheltered region. For the sheltered region its effect is mainly on the drag coefficient. $C_{D}$ varies, for thick boundary layers, approximately proportional to $(h / \delta)^{2 / 7}$ in the case of an infinitely wide shelter. For a finite 
width shelter, the effect should be even smaller, and thus, if we just make the profile approaching the shelter roughly logarithmic and as thick as possible, the values of $C_{D}$ obtained in the experiments should be transferable without much error to the atmospheric conditions, which leads to the proposed value of $C_{D} \approx 0.5-0.7$.

A sharp-edged shelter might be expected to shed regular eddies (of Kármán-type vortices), which would be the dominant feature in large-scale turbulence. On the other hand, flow through the porous screen may never allow significant lateral pressure excursions to occur such that an alternating structure would be observable. Recent measurements by Castro (1971) of wake characteristics of two-dimensional perforated plates in a uniform stream indicate that at an open area ratio of about 0.2 there is a radical change in the flow structure. Above a porosity of 0.2 apparently the formation of vortices ceases altogether and the energy thus preserved reflects in an abrupt reduction in drag coefficient. One may perform a similar analysis to that of Eq. (2) for the ratio of drags of a porous rectangular plate to a solid rectangular plate in a shear flow as compared with the ratio of two-dimensional porous plates to a solid plate in a uniform stream. A value of $C_{D}$ for the bug screen material of 0.16 is found which agrees exactly with the prediction of (8a). Indeed, even for solid prisms in a turbulent shear flow, the presence of regular shedding is indefinite $\left(\mathrm{Cermak}^{2}\right)$. Jensen and Franck (1963) remark from experiments of their own on wind screens of large aspect ratio that behind porous surfaces the vortex layer will certainly oscillate somewhat, all discontinuity surfaces being unstable. However, they observed that these small motions will cause only minute variations in the velocities. Nevertheless, one might wish to make an estimate of the likelihood of resonance of any gusts generated and a filling balloon. Vickery (1968) did obtain load fluctuation results for prisms in uniform turbulent flow. Thus, the frequency $f$ of the dominant eddies may be given approximately by the Strouhal frequency obtained from the relation

$$
\mathrm{St}=\frac{f W}{u_{\infty}}=0.08-0.11
$$

where St is Strouhal number, which according to results of Vickery is approximately constant and lies within the indicated range, and $f$ is the peak frequency. Typically, for a shelter $70 \mathrm{ft}$ in width, one would expect a dominant frequency of about (at $30 \mathrm{ft} \mathrm{sec}^{-1}$ )

$$
f=\frac{u_{\infty}}{W} \mathrm{St}=\frac{30}{70}(0.1) \approx 0.45 \mathrm{~Hz}
$$

For the $1 \mathrm{ft}$ wide model examined in the wind tunnel, a similar calculation would suggest a dominant fre-

\footnotetext{
${ }^{2}$ Cermak, J. E., 1971, private communication.
}

quency of about (at $30 \mathrm{ft} \mathrm{sec}^{-1}$ )

$$
f=\frac{30}{1}(0.1) \approx 3 \mathrm{~Hz} \text {. }
$$

Unfortunately this value is at the lower range of reliability for wind tunnel instrumentation and may not be readily apparent.

\section{Description of experiments}

\section{a. Wind tunnel simulation}

Wind tunnel measurements were performed in the Meteorological Wind Tunnel of the Fluid Dynamic and Diffusion Laboratory, Colorado State University. This tunnel has a $6 \mathrm{ft} \times 6 \mathrm{ft} \times 80 \mathrm{ft}$ long test section and generates a shear layer of the order of 2-3 ft thick (Plate and Cermak, 1963). Four basic shapes were tested; one consisted of a square plate set perpendicular to the wind $(1 \mathrm{ft} \times 1 \mathrm{ft})$ at a scale of $1: 70$, and the other three were of a wedge-type of same height and projection on the plane normal to the flow with apex angles of $90^{\circ}, 120^{\circ}$ and $150^{\circ}$. The models consisted of steel frames over which the screen materials had been stretched, as shown in Fig. 2c. They were designed with sharp outer edges, so that separation would always occur at the edges. Two different screen materials were tested: ordinary fiberglass bug screen $(0.010$ inch wire, 18 mesh; $\sim 0.67$ open area) and a special dense mesh fiberglass material provided by NCAR $(\sim 0.15$ open area). ${ }^{3}$

The presence of a partially inflated balloon was simulated by a rigid "ice-cream cone" shaped wooden model. The model had a $\frac{3}{4}$-inch diameter at the base and tapered to a 4 -inch diameter hemispherical cap. The model was, of course, not compliant to gust effects and stood a total of 10 inches high (see Fig. 4).

Vertical distributions of horizontal mean velocities were taken to map out the sheltered region. Using a coordinate system where $X$ is the distance downstream measured from the shelter trailing edge, $Y$ is the transverse coordinate measured from the screen centerline and $Z$ is in the vertical direction, velocity measurements were taken for $X=0(3) 18, Y=0(3) 12$ and $Z=0(5) 15$ inches (only one side of the shelter need be considered in view of the symmetry of the shelter about the $X Z$ plane). Velocities were obtained using a pitotstatic tube with a Transonic pressure transducer. A free-stream velocity range of $20-50 \mathrm{ft} \mathrm{sec}^{-1}$ was used on the four different shelters.

A gross measure of the tendency of the balloon shelter to dissipate the kinetic energy of the unrestrained wind is the turbulence intensity $\overline{u^{\prime 2}}$, where $u^{\prime}$ is the fluctuating velocity component (with time mean zero) in the direction of the mean local flow velocity. The

${ }^{3}$ Style $9710-18 \times 56$ mesh fiberglass cloth screen manufactured by J. P. Stevens and Co., Watterboro, S. C. 


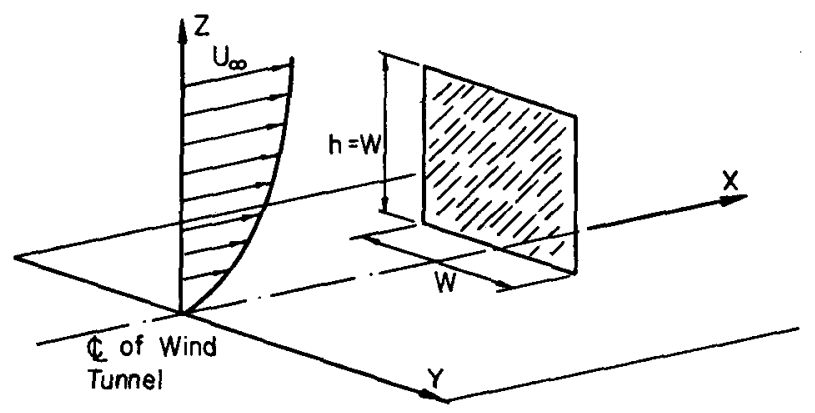

A Schematic of Square Screen

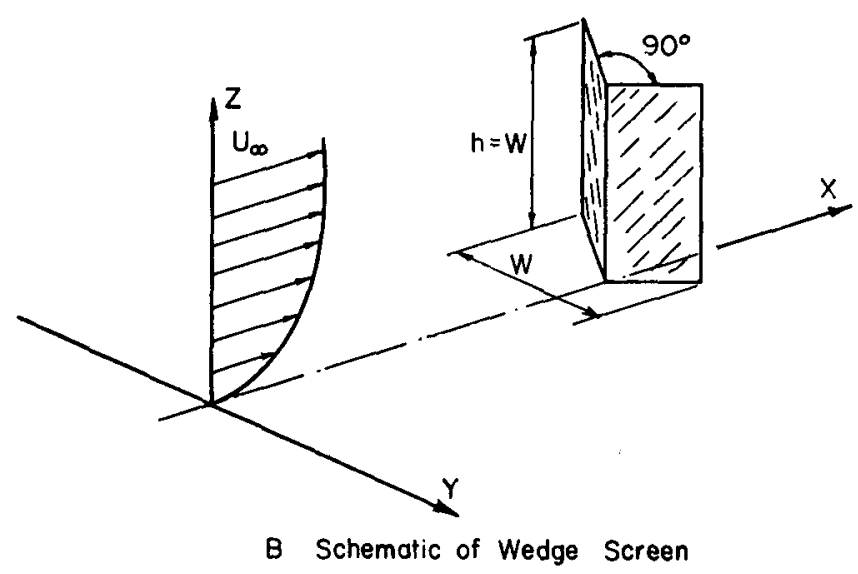

FIG. 2. Screen models.

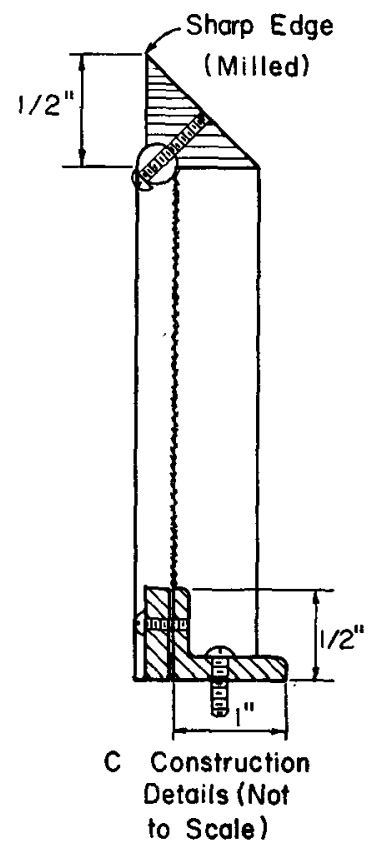

overbar denotes the time mean. Due to the limitations of the rms-analyzers utilized, these data are of frequencies higher than $2 \mathrm{cps}$ and are thus not representative of the low-frequency end of the spectrum, which is of greatest importance for balloon sheltering. It also became clear that one area of major interest was the side edges of a shelter. Further examination of this area involved measuring turbulence intensities and making frequency analyses of the eddy shedding at the edges. Turbulence intensities were measured using a Disa constant temperature anemometer, type 55A01.

Two types of turbulence spectral data were obtained: pressure fluctuations of a pitot-static tube recording at a distance of 3 inches from the centerline at four different downstream distances of the NCAR screen square plate and wedge, at one height of 6 inches $\left(=\frac{1}{2} h\right)$ above the floor. These data, recorded on strip charting, give an ndication of the low-frequency turbulence which is likely to affect the balloons. However, one cannot detect any low-frequency component in the recordings which might be significant. A second set of turbulence data was obtained from the output signal of a constant temperature hot wire anemometer. Special attention was given to the shear flow at the shelter edge. The signal from the anemometer was analyzed using General Radio's Graphic Level Recorder type 1510-A, coupled to a Sound and Vibration Analyzer, type 1911-A. This equipment provides a frequency response from 2.5 to $25 \mathrm{~Hz}$.

Pressure drop coefficients $c_{p}$ were obtained by stretching screens across the whole cross section of a small duct, and measuring velocity and pressure drop across the screen with two pitot-static tubes located one upstream and one downstream of the screen. For the NCAR screen, we found a pressure drop coefficient $c_{p}$ of 22-implying an almost solid screen--independent of 
Re. For the bug screen, the pressure drop coefficient was found to be 0.62 . For a bug screen prepared in a double layer with $45^{\circ}$ angle between the mesh orientation, the pressure drop coefficient was about 1.25. Again, all Reynolds number dependencies, if existing, were hidden in the scatter of the experimental results. These values of pressure coefficients agree qualitatively with the estimating expressions suggested by Hoerner (1965). For a screen of high solidity $\sigma$, he recommends $c_{p}=[\sigma /(1-\sigma)]^{2}$ and for low solidity, $\sigma<0.5, c_{p}=C_{D} /$ $(1-\sigma)$, where $C_{D}$ is the drag coefficient of the screen element. For the dense NCAR screen $(\sigma=0.85)$ and for the open bug screen ( $\sigma=0.33$, wire elements) one obtains $c_{p} \approx 32$ and 0.73 , respectively, which are the order of those measured.

\section{b. Wind-screen field tests}

A 1:7 scale model of the proposed shelter was constructed by E.G.\&G. Inc. of Boulder, Colo., and tested under the direction of NCAR at Marshall Field south of Boulder (Fig. 3). The balloon shelter was constructed by stretching fiberglass bug screen over a frame of 2 -inch $\times 4$-inch wooden boards. The shelter consisted of two flat sections measuring $10 \mathrm{ft}$ high by $5.75 \mathrm{ft}$ wide, hinged together on a $10 \mathrm{ft}$ edge. When standing with an apex angle of $120^{\circ}$, the shelter presents a $10 \mathrm{ft} \times 10 \mathrm{ft}$ frontal area to the impending wind.

Measurements were made on a day with ambient winds in the 10-20 kt range. The shelter was erected with the apex pointing into the wind. Reference cup anemometers were located $40 \mathrm{ft}$ upwind of the shelter, $5 \mathrm{ft}$ above the ground, and $28 \mathrm{ft}$ downwind of the shelter, $5 \mathrm{ft}$ above the ground. Data were continuously monitored from these sensors as a mid-wind sensor was placed in various regions of the sheltered zone. The mid-wind sensor was operated both at $5 \mathrm{ft}$ and at 9 feet 4 inches above the ground, first with two layers of bug screen attached and then with one layer. Data were recorded for at least $2 \mathrm{~min}$ for each mid-wind location, height, and layer of the screen.

\section{Results and discussion}

\section{a. Wind tunnel simulation}

To obtain an estimate of the amount of flow deflected around the shelters smoke tracers were released upstream of the shelters. For very low free-stream velocities the majority of the smoke was deflected right along the upstream face. For higher free-stream velocities, most of the smoke passed through the screen about halfway along the screen surface. With the balloon model placed behind the screen the smoke pattern was not observably changed (Fig. 4).

The other visualization technique involved looking at the motion of a small cork ball which was attached by thread to a long wire rod. Holding the wire rod from outside the flow field, the ball could be located at

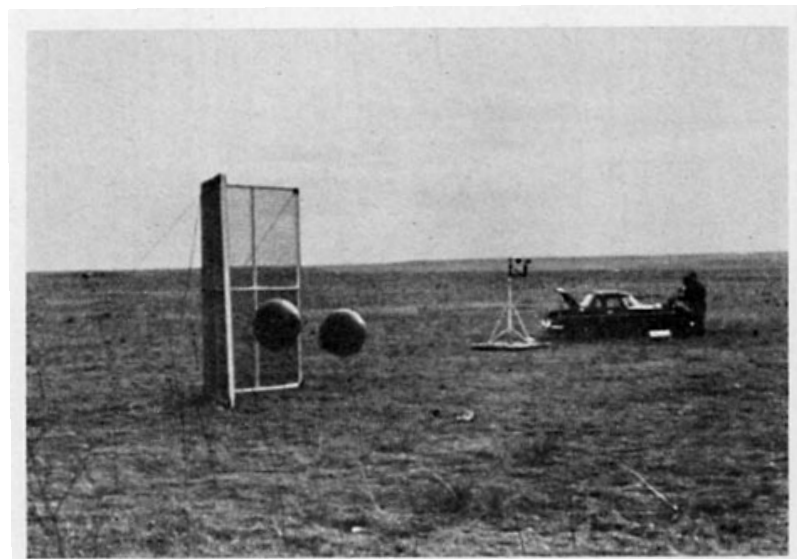

FIG. 3. Wind screen erected at Marshall Field site, Boulder, Colo.

positions about the shelter. The areas of main interest were the shelter sides and top edges. No rotation of the ball was observed over the top edges; however, at the side edges the ball rotated vigorously. As the ball was drawn across a vertical side support (in the direction of decreasing $Y$ ), the rapid rotation of the ball abruptly reversed. A short distance inside the support (that is, in the sheltered region) the ball rotation slowed and ceased. It is felt that this vortex phenomenon at the shelter edges is due entirely to the vertical supports.

A typical profile of the approach velocity for the shelters is shown in Fig. 5 at a distance of $5 w$ upstream of the model. From the complete set of such profiles isotachs were constructed. Two typical types of figures were prepared (Figs. 6-9). Profiles along the centerline, to show the reduction of wind velocity along this plane, and cross sections through the sheltered regions were prepared. Downwind distances from the wedges are measured from the downwind edges of the model.

From the techniques described, it appears that all shelter angles give approximately the same sheltered region with a velocity reduction of about $50 \%$. Eddy

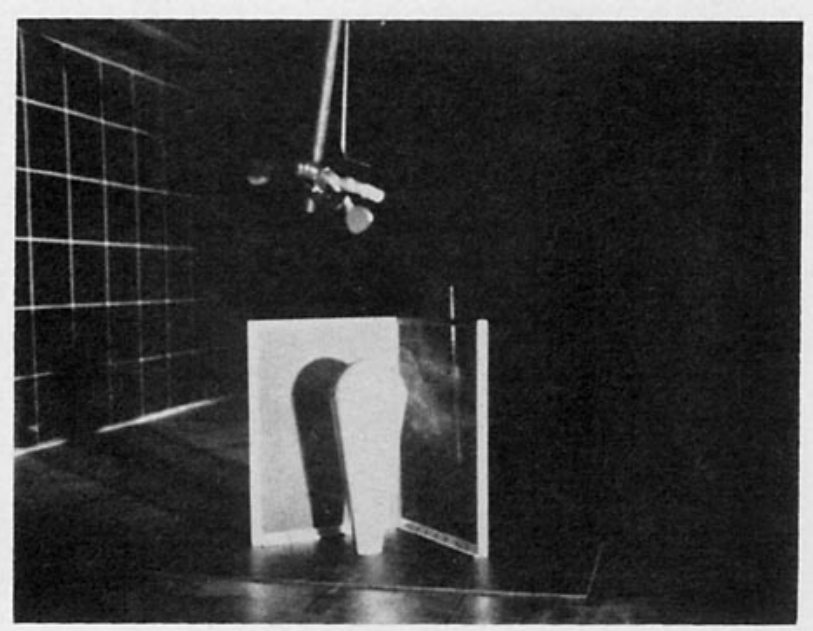

FIG. 4. Smoke visualization past $120^{\circ}$ wedge with balloon model: $U_{\infty} \approx 5 \mathrm{ft} \mathrm{sec}^{-1}$. 


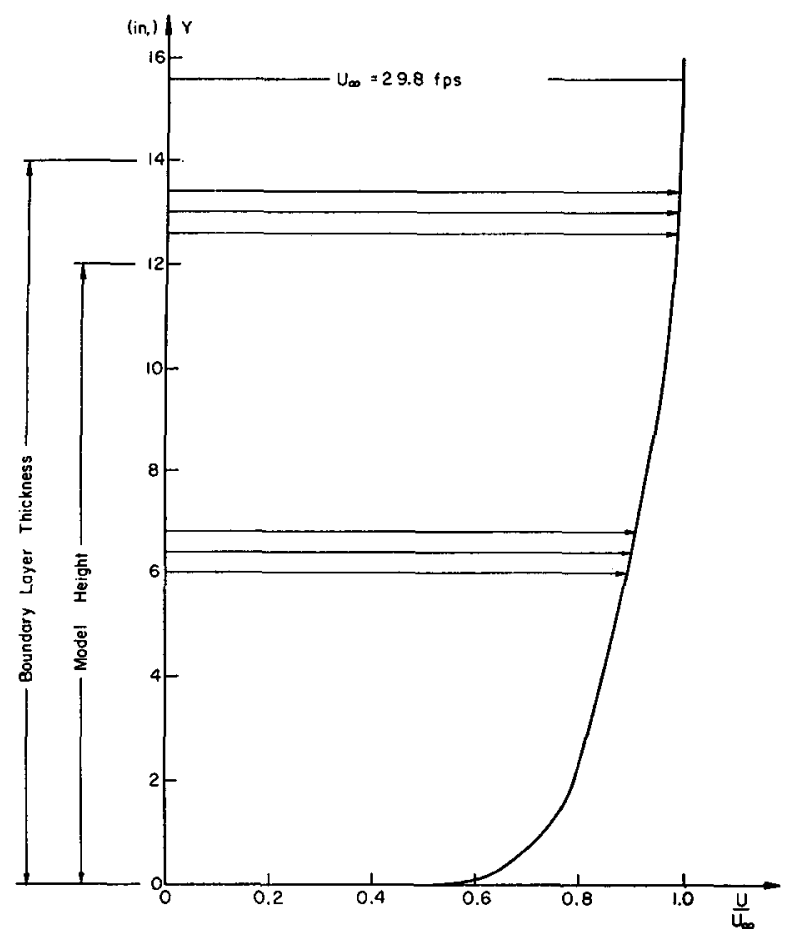

Fig. 5. Approach wind profile (at $X=5 w$ upstream of model).

shedding from the shelter supports is an important feature but is confined to the side edges of the shelter and does not affect the sheltered area. No other largescale vortices related to the shelter geometry were observed.

With the wooden balloon model in place a marked decrease in velocity on the centerline with an increase around the sides and over the top of the balloon was observed. A short distance downstream these two effects seemed to combine to produce a flow pattern similar to that of the "no balloon" case.

Using the Disa hot-wire anemometer, the turbulence intensity distribution around the shelters was investigated. The free-stream turbulence level was about $3 \%$. Behind the screen section of a shelter the turbulence level was very low $(4 \%)$, but on the centerline (i.e., behind the center support) the level rose to $20 \%$. The effect was most marked at the edges, $40 \%$ intensity being the general value. These high values at the edges are consistent with the vortex ball investigation. The vortex ball, however, failed to indicate the relatively high turbulence level due to the center support. Typical profiles of $\overline{u^{\prime 2}}$ along a distance $1 / 4 w$ off the centerline are shown in Fig. 10.

The signal from the anemometer was subjected to a frequency spectrum analysis, the eddy shedding frequency at the edges being the major area of interest. The possible frequency for dominant eddies, given approximately by the Strouhal frequency, was of the order $10 \mathrm{~Hz}$. Extensive investigation failed to isolate this frequency. In addition to the arguments cited previ-
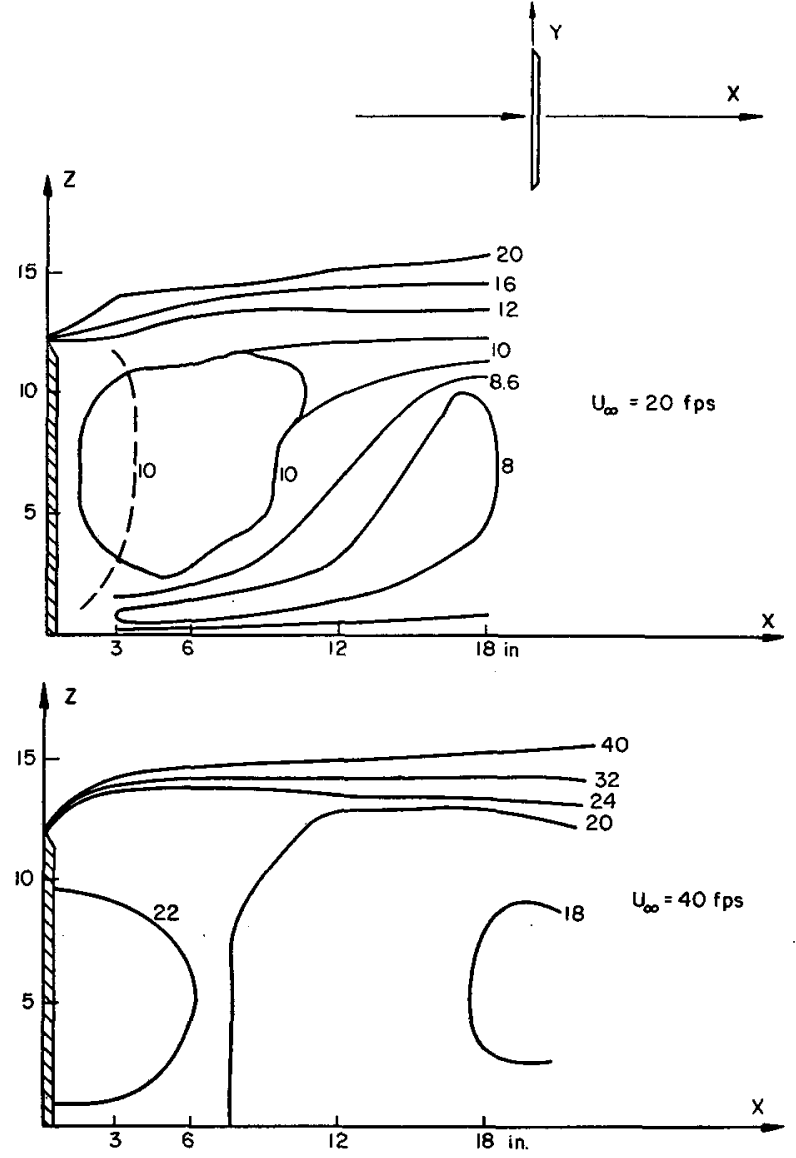

FIG. 6. Centerline velocity isotachs behind square plate screen (porosity $\sim 0.67$ ).
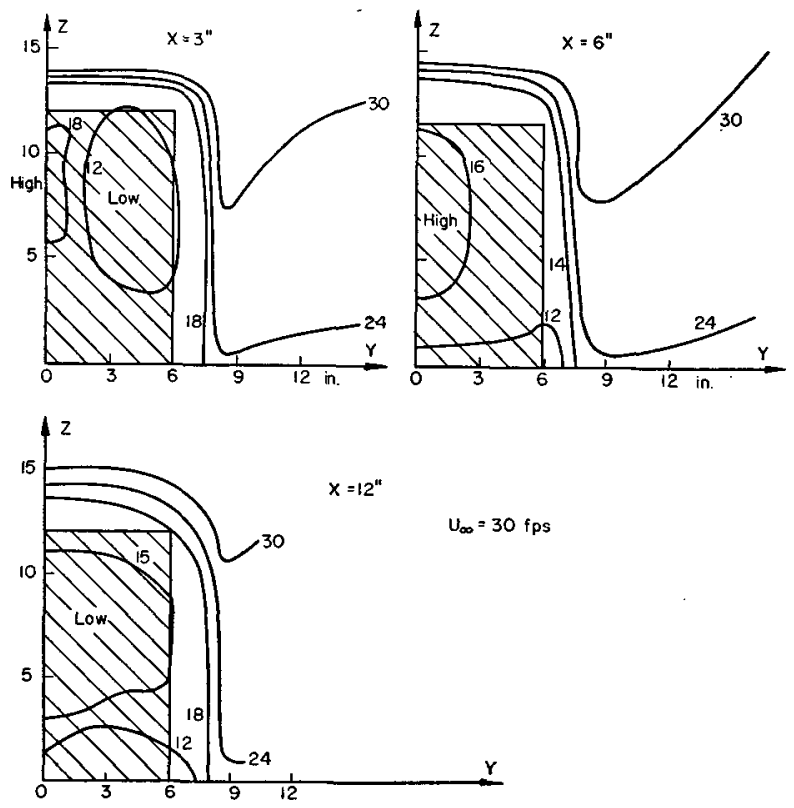

FIg. 7. Cross section velocity isotachs behind square plate bug screen (porosity $\sim 0.67$ ). 

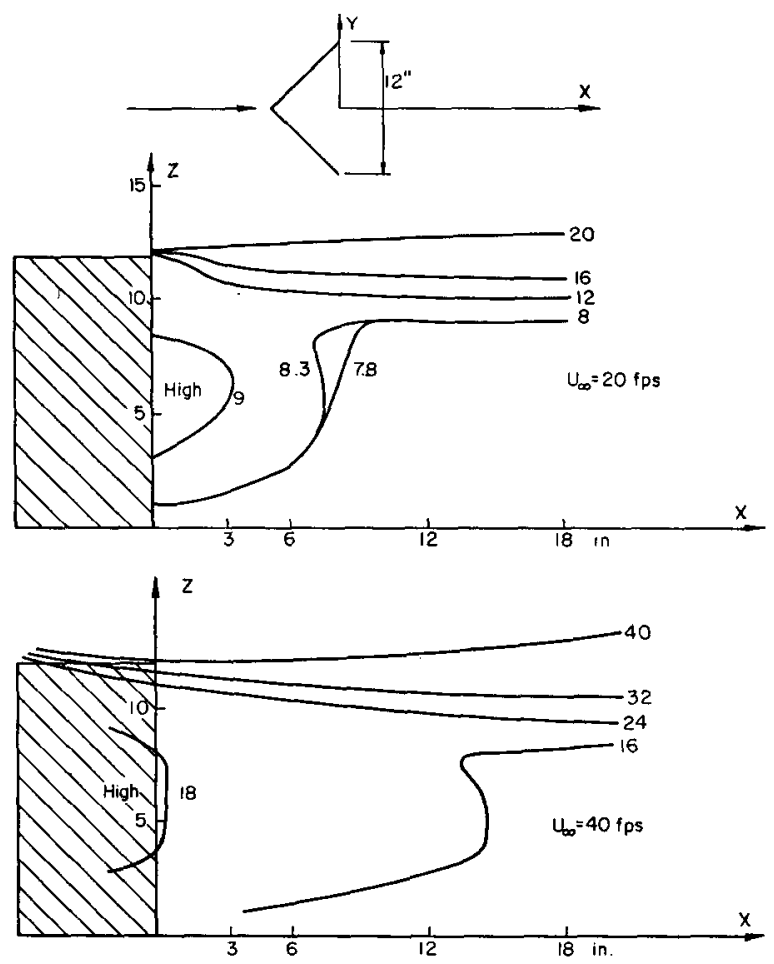

FIG. 8. Centerline velocity isotachs behind $90^{\circ}$ wedge screen (porosity $\sim 0.67$ ).

ously, there is the possibility that for porous structures the realm of regular frequencies ceases for $\operatorname{Re}>10^{5}$. Indeed, even for cylinders measurements for high $\mathrm{Re}$ numbers are very sparse. Roshko has suggested a Strouhal gap exists between $10^{5}$ and $10^{7}$ for eddy
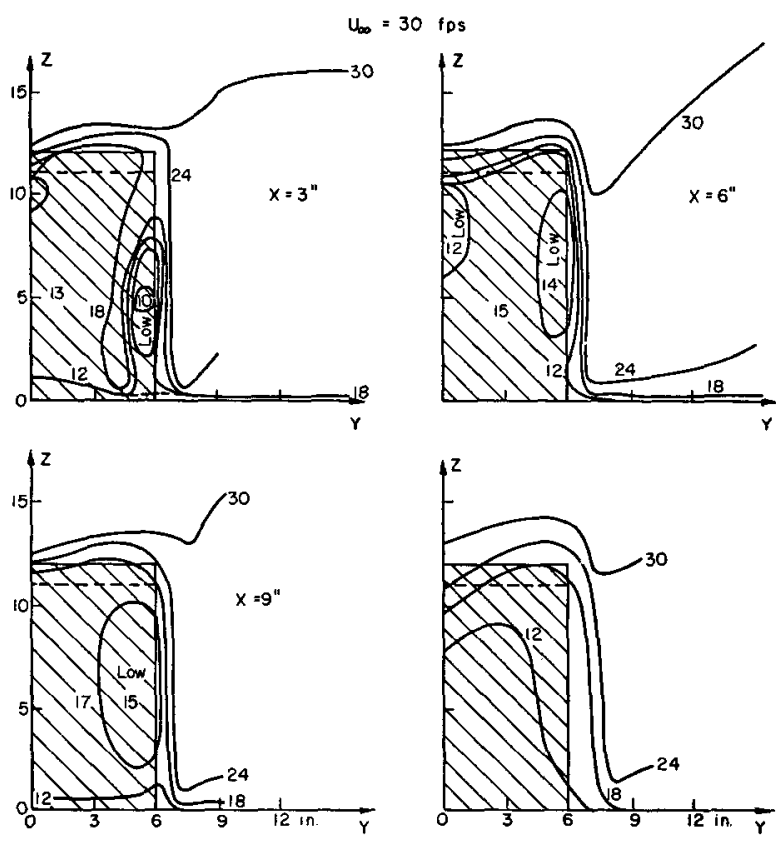

Fig. 9. Cross section velocity isotachs behind $90^{\circ}$ wedge screen (porosity $\sim 0.67$ ) $U_{\infty}=30 \mathrm{ft} \mathrm{sec}-1$. shedding from cylinders (Morkovin, 1964). Though the Re number for the prototype shelter size may well be greater than $10^{7}$, one may have difficulty in identifying a specific maximum shedding frequency since 1) the maximum becomes increasingly blurred at high $\mathrm{Re}$ number, and 2) the flow through the porous screen may never allow significant lateral pressure excursions to occur such that an alternating structure may be observable.

The general character of velocity reduction behind the shelters for the different configurations was discussed above. For the single screen shelter the velocity reduction for all angles was over $50 \%$. For the double screen shelter the reduction was about $75 \%$ with the pressure coefficient increasing to twice that of the single screen case.

Two dimensionless parameters, $\xi$, a mass flux parameter and $\psi$, a momentum flux parameter, were defined; i.e.,

$$
\begin{aligned}
& \xi=\int_{0}^{y} \rho u d y / \rho U_{\infty} L, \\
& \psi=\int_{0}^{y} \rho u\left(U_{\infty}-u\right) d y / \rho U_{\infty}^{2} L
\end{aligned}
$$

where $u$ is the velocity behind the shelter, $U_{\infty}$ the freestream velocity and $L$ the total width of the shelter (12 inches). The momentum flux parameter $\psi$ may be
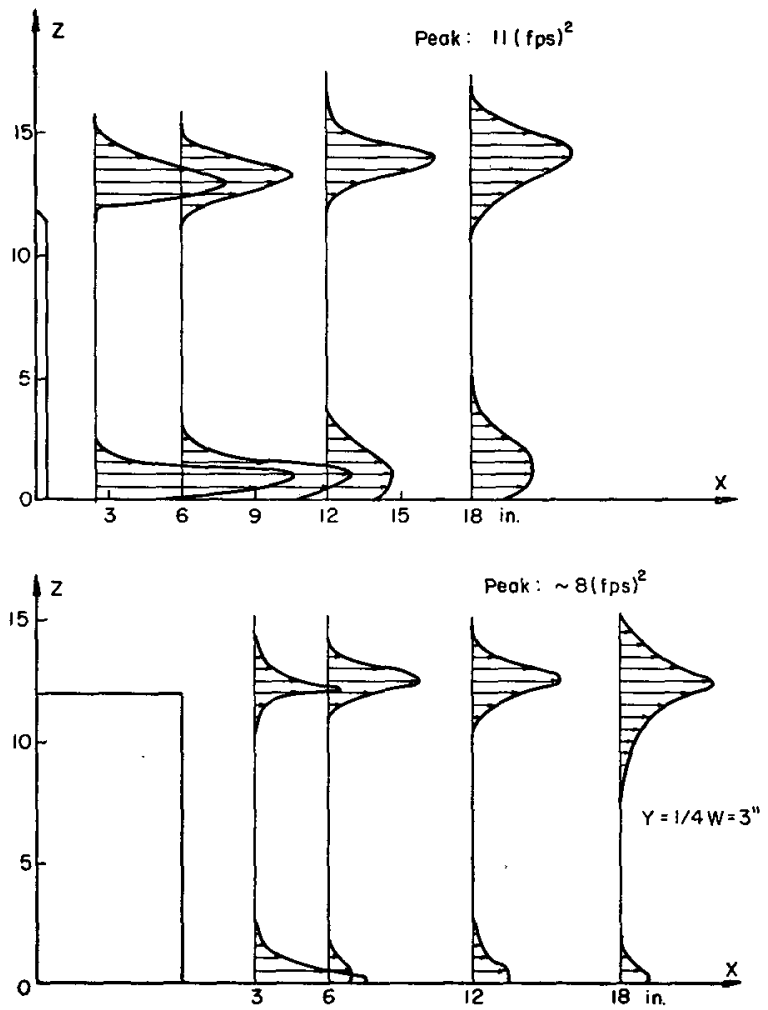

Fig. 10. Turbulent intensity profiles downstream of square and $90^{\circ}$ wedge screens (porosity $\sim 0.15$ ). 
TABLe 1. Mass flux parameter $\xi$ (dimensionless), calculated for various free-stream velocities at $Z / L=5 / 6$ and for various $X / L$ coordinates.

\begin{tabular}{|c|c|c|c|c|}
\hline $\begin{array}{c}U_{\infty} \\
\left(\mathrm{ft} \sec ^{-1}\right)\end{array}$ & $\frac{X}{L}=\frac{1}{4}$ & $\frac{X}{L}=\frac{1}{2}$ & $\frac{X}{L}=1$ & $\frac{X}{L}=\frac{5}{4}$ \\
\hline \multicolumn{5}{|c|}{$90^{\circ}$ shelter (no balloon) } \\
\hline $\begin{array}{l}19.0 \\
31.5 \\
48.6\end{array}$ & $\begin{array}{l}0.48 \\
0.54 \\
0.52\end{array}$ & $\begin{array}{l}0.57 \\
0.61 \\
0.59\end{array}$ & $\begin{array}{l}0.58 \\
0.65 \\
0.59\end{array}$ & $\begin{array}{l}0.61 \\
0.65 \\
0.61\end{array}$ \\
\hline \multicolumn{5}{|c|}{$90^{\circ}$ shelter (with balloon) } \\
\hline 19.0 & 0.64 & 0.63 & 0.62 & \\
\hline \multicolumn{5}{|c|}{$120^{\circ}$ shelter (no balloon) } \\
\hline 19.0 & 0.46 & 0.51 & 0.51 & 0.50 \\
\hline \multicolumn{5}{|c|}{$120^{\circ}$ double screen shelter (no balloon) } \\
\hline $\begin{array}{l}19.0 \\
31.5 \\
48.6\end{array}$ & $\begin{array}{l}0.35 \\
0.36 \\
0.41\end{array}$ & $\begin{array}{l}0.38 \\
0.40 \\
0.39\end{array}$ & $\begin{array}{l}0.38 \\
0.37 \\
0.37\end{array}$ & $\begin{array}{l}0.35 \\
0.35 \\
0.35\end{array}$ \\
\hline \multicolumn{5}{|c|}{$150^{\circ}$ shelter (no balloon) } \\
\hline 19.0 & 0.45 & 0.50 & 0.52 & \\
\hline \multicolumn{5}{|c|}{$150^{\circ}$ shelter (with balloon) } \\
\hline 19.0 & & 0.61 & 0.59 & 0.58 \\
\hline
\end{tabular}

viewed as a pseudo-drag coefficient in the sense that it is a measure of the blocking effect of the shelter. It is, of course, not exactly a drag coefficient, since the fluid motion is three-dimensional and corrections must be made for static pressure variations when the traverse is close to the shelter. Schlichting (1968) discusses correction methods to be applied to drag calculations from measurements in the mean wake vicinity. These

TABLE 2. Momentum flux parameter $\psi$ (dimensionless), calculated for various free-stream velocities at $Z / L=5 / 6$ and for various $X / L$ coordinates.

\begin{tabular}{|c|c|c|c|}
\hline $\begin{array}{c}U_{\infty} \\
\left(\mathrm{ft} \sec ^{-1}\right)\end{array}$ & $\frac{X}{L}=\frac{1}{2}$ & $\frac{X}{L}=1$ & $X=\frac{5}{4}$ \\
\hline \multicolumn{4}{|c|}{$90^{\circ}$ sheiter (no balloon) } \\
\hline $\begin{array}{l}19.0 \\
31.5 \\
48.6\end{array}$ & $\begin{array}{l}0.21 \\
{ }^{\circ} \text { shelt }\end{array}$ & $\begin{array}{c}0.21 \\
0.21 \\
0.23 \\
\text { balloo }\end{array}$ & $\begin{array}{l}0.22 \\
0.21 \\
0.22\end{array}$ \\
\hline 19.0 & 0.21 & 0.21 & \\
\hline \multicolumn{4}{|c|}{$120^{\circ}$ shelter (no balloon) } \\
\hline 19.0 & & 0.21 & 0.22 \\
\hline \multicolumn{4}{|c|}{$120^{\circ}$ double screen shelter (no balloon) } \\
\hline $\begin{array}{l}19.0 \\
31.5 \\
48.6\end{array}$ & & $\begin{array}{l}0.17 \\
0.19 \\
0.19\end{array}$ & $\begin{array}{l}0.18 \\
0.18 \\
0.18\end{array}$ \\
\hline \multicolumn{4}{|c|}{$150^{\circ}$ shelter (no balloon) } \\
\hline 19.0 & 0.21 & 0.21 & \\
\hline \multicolumn{4}{|c|}{$150^{\circ}$ shelter (with balloon) } \\
\hline 19.0 & 0.20 & 0.21 & 0.21 \\
\hline
\end{tabular}

parameters were calculated for the wake of the different shelters and are tabulated in Tables 1 and 2. It can be seen that there is little variation in the parameters over the set of single screen shelters suggesting that shelter shape has little effect on the downstream region. In the double screen case, the decrease in these parameters is consistent with our intuition. Again little variation is seen over the range of free-stream velocities.

Intuition suggests that shelter angle should influence the sheltered zone since the wind must approach the screen at an oblique angle resulting in a decreased effective permeability. Eimern (1964) reports the results of numerous investigators concerned with wind break effectiveness at oblique angles of wind incidence. Although a number of authors report marked changes when wind angles exceed $30^{\circ}$, others finally assert that variations in angle of incidence to $45^{\circ}$ cause no important change in sheltering. Again any suggested influence is in the extent of the sheltered zone rather than its quality; hence, one concludes the absence of effect of shelter shape is not abnormal for this study.

\section{b. Wind screen field test}

Wind velocity ratios were removed from the records of the field experiment and compared with the wind tunnel tests. Ten of the fourteen measuring sites in the field were directly comparable to the wind tunnel results. The time varying wind records were averaged and standard deviations calculated. The general shape of the sheltered zones were equivalent. The single screen, $120^{\circ}$ apex angle test reduced the velocity by $\sim 0.35$ with a variance of $\sim 0.025$. The double screen, $120^{\circ}$ apex angle test reduced the velocity by $\sim 0.50$ with a variance of $\sim 0.035$. The instantaneous upstream to mid-region velocity ratio varied from $0.16-1.0$ and $0.07-1.0$ for the single and double screen cases, respectively. This wide variation may result from wind veering or the lack of spatial correlation of velocity between anemometers.

The field measurements, in general, did not reveal as large a sheltering effect as the wind tunnel measurements. The single screen provided $50 \%$ reduction in the wind tunnel as compared with $35 \%$ reduction in the field. The double screen performed at $75 \%$ and $50 \%$ reduction levels, respectively. The proportionate increase in the added shelter of the doubled screen was about the same for both experiments.

\section{Conclusions}

On the basis of the reported experiments, the following conclusions on the design of a balloon shelter are drawn:

1) Porous shelter surfaces, as compared to solid (or almost solid surfaces), have a considerably lower turbulence level associated with them, but a mean velocity level which is higher in the sheltered region. Furthermore, the forces on a porous screen are much smaller. A rough estimate gave drag coefficients for the square 
plate data of $0.5-0.7$, and 0.16 , for solid and bug screen surfaces, respectively.

2) The shelter angle has no noticeable effect on velocity reduction, turbulence level or flow pattern.

3) Velocity reduction for all angles with single screen is over $50 \%$ with $75 \%$ reduction in the double screen case. The pressure coefficient is doubled for the double screen case. Neither the flow pattern nor the percentage reductions attained depended on the ambient velocity $U_{\infty}$. Consequently, it is felt that prototype screen and model screens should be the same. It is recommended that a material should be used for the screens which is slightly denser than the bug screen, such as a double layer of bug screen or equivalent.

4) The balloon presence produces higher velocities around the balloon surface. Downstream the flow pattern returns to the "no balloon" case.

5) Eddy shedding from the structure's edges could interact with the balloon if the shelter was too narrow.

6) A blockage near the structure may occur due to the vertical side supports. The velocity decrease behind these supports recovers quickly with distance downstream.

7) A square plate shelter provides a larger sheltered area, but a more intense turbulence intensity than a wedge-shaped design. On this basis, and on the basis of construction convenience, it is recommended that the wedge be used, in a suitable modification to meet structural requirements.

\section{REFERENCES}

Castro, I. P., 1971: Wake characteristics of two-dimensional perforated plates normal to an airstream. J. Fluid Mech., 46, 599-609.

E.G.\&G., 1971: Report on balloon shelter tests. Prepared for National Center for Atmospheric Research, under Purchase Order NCAR 156-70, to E.G.\&G., Inc., Environmental Services Operation, Boulder, Colo.

Eimern, J. van, 1964: Windbreaks and shelterbelts. World Meteorological Organization, Tech. Note No. 59, Geneva, 188 pp.

Hoerner, S. F., 1965: Fluid Dynamic Drag. Midland Park, N. J., published by the author, $450 \mathrm{pp}$.

Jensen, M., and N. Franck, 1963: Model Scale Tests in Turbulent Wind. Copenhagen, Danish Technical Press, $97 \mathrm{pp}$.

Morkovin, M. V., 1964; Flow around circular cylinder-A kaleidoscope of challenging fluid phenomena. Proc. Symp. on Fully Separated Flow, ASME, 18-20 May 1964, 102-118.

Plate, E., 1964: The drag on a smooth flat plate with a fence immersed in its turbulent boundary layer. ASME Paper No. 64 FE-17.

- - , and J. E. Cermak, 1963: Micrometeorological wind-tunnel facility: Description and characteristics, Fluid Dynamic and Diffusion Laboratory, Tech. Rept. CER63EJP-JEC9, Colorado State University, Fort Collins.

—, and C. Y. Lin, 1965: The velocity field downstream from a two-dimensional model hill. Final Report, Part I, U. S. Army Material Agency, Contract DA-AMC-36-039-63-G7.

Rouse, H., 1950: Engineering Hydraulics. New York, Wiley, $1039 \mathrm{pp}$.

Schlichting, H., 1968: Boundary Layer Theory. New York, McGraw-Hill, $747 \mathrm{pp}$.

Vickery, B. J., 1968: Load fluctuations in turbulent flow. Proc. ASCE, J. Engr. Mech. Div., 94, Paper No. Eml. 